\title{
Spectroscopic Diagnostic of Helium-Hydrogen RF Plasma under the Influence of Radiation Trapping
}

\author{
Keiji SAWADA, Motoshi GOTO ${ }^{1)}$ and Naomichi EZUMI ${ }^{2)}$ \\ Faculty of Engineering, Shinshu University, 4-17-1 Wakasato, Nagano 380-8553, Japan \\ 1) National Institute for Fusion Science, 322-6 Oroshi-cho, Toki 509-5292, Japan \\ ${ }^{2)}$ Nagano National College of Technology, 716 Tokuma, Nagano 381-8550, Japan
}

(Received 18 May 2010 / Accepted 26 December 2010)

\begin{abstract}
The electron temperature and density, atomic hydrogen density and temperature in a helium-hydrogen RF plasma are determined from the visible emission line intensities of both atoms by considering photoexcitation from the ground state accompanied by radiation trapping in the plasma. From the observed helium line intensity, and the hydrogen Balmer $\gamma$ line intensity which is little affected by photoexcitation, parameters other than the atomic hydrogen temperature are determined using a helium atom collisional-radiative model [Sawada et al., Plasma Fusion Res. 5, 001 (2010)], which includes photoexcitation for helium singlet P states, and a hydrogen atom collisional-radiative model in which photoexcitation is ignored. The atomic hydrogen temperature is determined to reproduce the Balmer $\alpha$ and $\beta$ line intensities by using an iterative hydrogen atom collisional-radiative model [Sawada, J. Plasma Phys. 72, 1025 (2006)] that calculates the photoexcitation rates.
\end{abstract}

(C) 2011 The Japan Society of Plasma Science and Nuclear Fusion Research

Keywords: radiation trapping, helium-hydrogen plasma, collisional-radiative model, spectroscopic diagnostic

DOI: $10.1585 /$ pfr.6.1401010

\section{Introduction}

In future fusion plasmas, helium will be produced by nuclear fusion of deuterium and tritium. In the study of edge plasmas, it is important to develop a diagnostic method to examine the emission lines of hydrogen isotope atoms and helium atoms.

Optically thin emission lines allow the local population of the upper states of the emission to be determined. However, in many cases, the population is generated by photoexcitation from the ground state due to radiation trapping in a plasma. Thus, it is essential to develop a model that includes radiation trapping and understand its contribution to emission intensity.

The emission or absorption of photons in a location depends on the radiation flux emitted from the rest of the plasma. Because atoms at a location influence each other through emission or absorption, a self-consistent treatment of radiation trapping is necessary for calculating the excited state population of the atoms. For hydrogen plasmas, an iterative self-consistent collisional-radiative model has been developed [1]. For helium plasmas, a method has been developed for evaluating the contribution of photoexcitation to the excited state populations by using the observed helium line intensities [2].

In this study, the intensities of atomic hydrogen and helium emission lines in an RF helium-hydrogen plasma located at Shinshu University in Japan are investigated. The purposes of this paper are to propose a method for de- termining the electron temperature and density and atomic hydrogen density by analyzing the observed helium and hydrogen line intensities simultaneously, and to develop a method for estimating atomic hydrogen temperature by evaluating the contribution of radiation trapping to the Balmer line intensities.

\section{Experimental}

Figure 1 shows a schematic diagram of the apparatus, which consists of glass tubes $5 \mathrm{~cm}$ in diameter. A base pressure of $9 \times 10^{-7}$ Torr was maintained in the glass tubes by a diffusion-rotary pump. A gaseous mixture of helium and hydrogen was introduced through mass flow controllers. The flows of helium and hydrogen gas were $450 \mathrm{sccm}$ and $20 \mathrm{sccm}$, respectively. The gas pressure, measured using a baratron gauge, was 0.144 Torr. The particle density estimated from the gas pressure by using the ideal gas law at room temperature $(300 \mathrm{~K})$ was

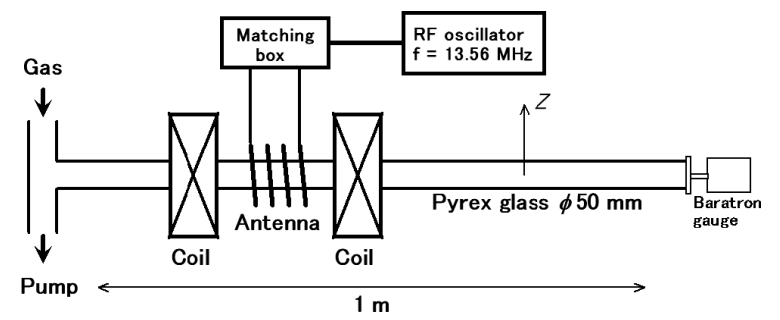

Fig. 1 Schematic representation of experimental apparatus. 

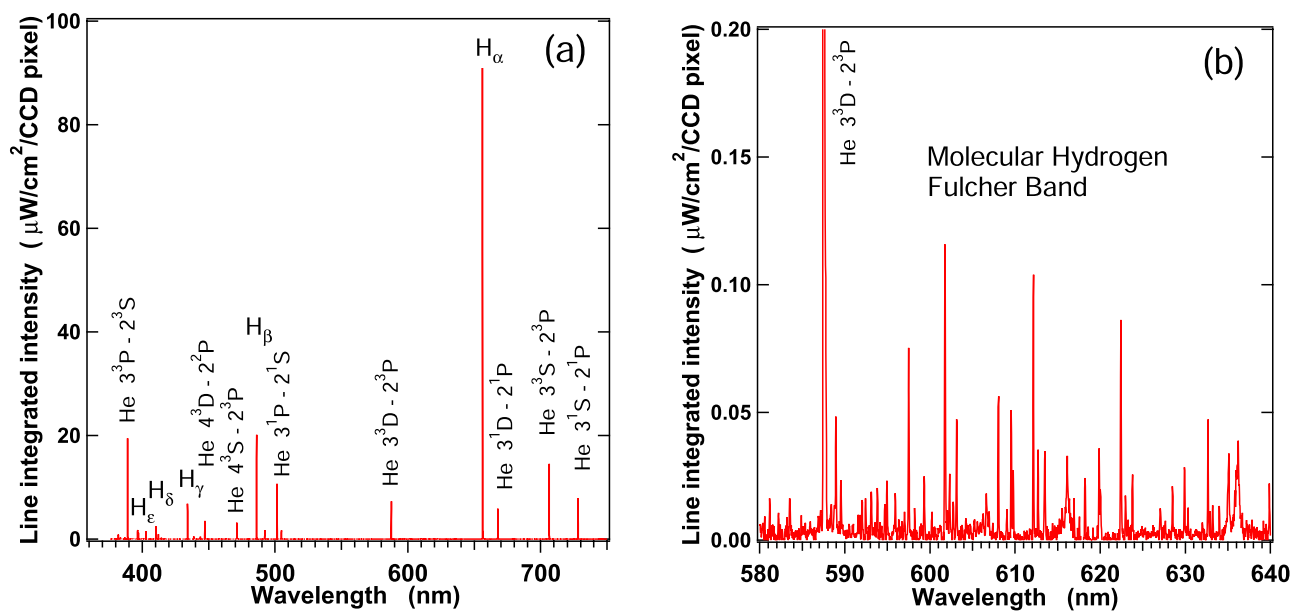

Fig. 2 (a) Spectrum observed with the echelle spectrometer. (b) Observed molecular hydrogen Fülcher band $\left(d^{3} \Pi_{u} \rightarrow a^{3} \Sigma_{g}^{+}\right)$spectrum. $Z=0 \mathrm{~cm}$.
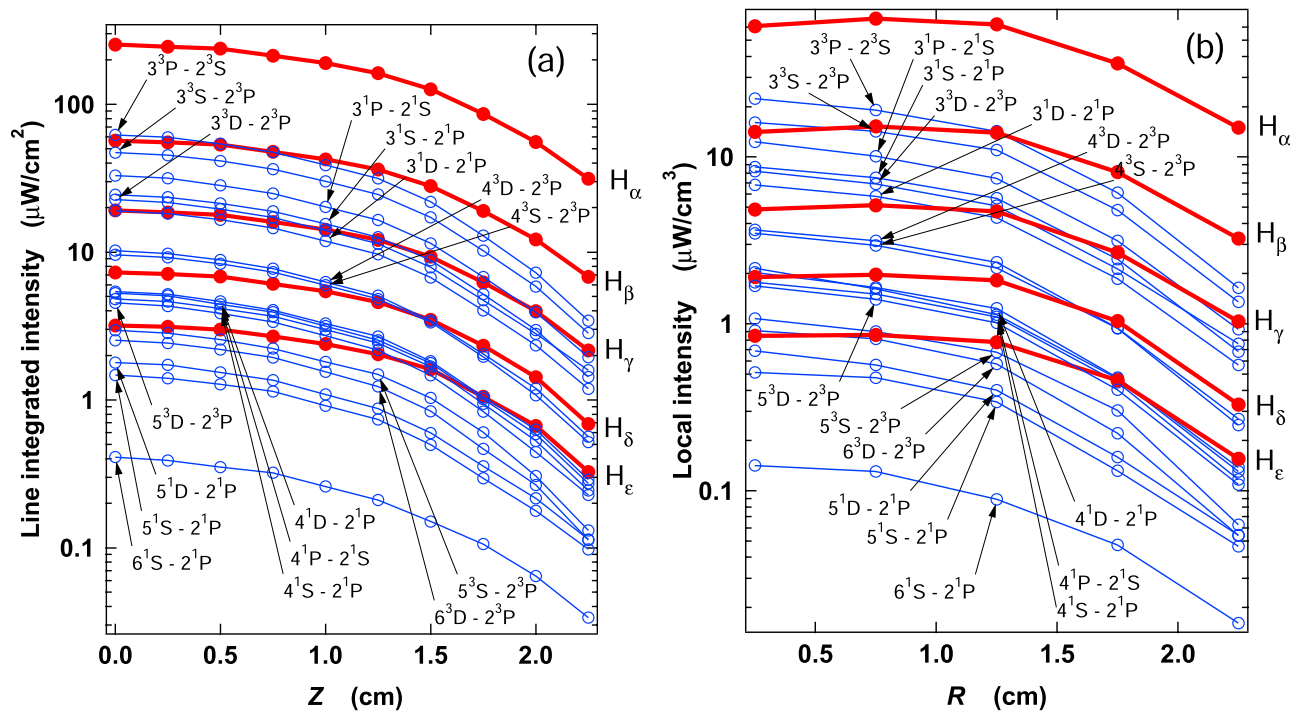

Fig. 3 (a) Line-of-sight integrated intensities. (b) Intensities as a function of plasma radius obtained by Abel inversion. Closed circles: atomic hydrogen Balmer series lines; open circles: atomic helium lines.

$4.6 \times 10^{15} \mathrm{~cm}^{-3}$. A water-cooled RF antenna connected to a matching network was then supplied with an RF power of $800 \mathrm{~W}$ at $13.56 \mathrm{MHz}$. A magnetic field of approximately $100 \mathrm{G}$ was produced around the antenna by a pair of solenoids. We measured the intensities of emission lines of atomic helium and hydrogen. The line of sight was scanned along the $Z$-axis in Fig. 1 by shifting a collecting lens. The collected light was fed via an optical fiber to an echelle spectrometer (BUNKOUKEIKI EMP200-AS) with a CCD camera (ANDOR DV420), which covers the wavelength range of $376-800 \mathrm{~nm}$. The optical fiber with the collecting lens provided a spot size of approximately $4 \mathrm{~mm}$. The absolute sensitivity of the optical system was calibrated using a calibrated xenon lamp light source (Hamamatsu L7810).

Figure 2 (a) shows an example of the measured spectra. Figure 2 (b) shows the observed molecular hydrogen
Fülcher band $\left(d^{3} \Pi_{u} \rightarrow a^{3} \Sigma_{g}^{+}\right)$spectrum, which will be used to estimate the molecular hydrogen density. Table 1 lists the identified emission lines, and Fig. 3 (a) shows the lineof-sight integrated intensities of the emission lines. Figure 3 (b) shows the local intensities derived using Abel inversion.

\section{Model}

First, we briefly summarize the model used in this study. Because the present RF plasma is an ionizing plasma [3], we describe the model of the ionizing plasma for simplicity.

\subsection{Collisional-radiative model for helium atom}

For atomic helium, a collisional-radiative model developed in Ref. [2,4-6] was used. The population of the 
Table 1 Identified atomic hydrogen Balmer series and atomic helium lines.

\begin{tabular}{cccc}
\hline \hline Atom & Transition & Wavelength $[\mathrm{nm}]$ & $A$ coefficient $\left[10^{8} \mathrm{~s}^{-1}\right]$ \\
\hline $\mathrm{H}$ & $2-7\left(\mathrm{H}_{\epsilon}\right)$ & 397.007 & 0.004389 \\
$\mathrm{H}$ & $2-6\left(\mathrm{H}_{\delta}\right)$ & 410.173 & 0.009732 \\
$\mathrm{H}$ & $2-5\left(\mathrm{H}_{\gamma}\right)$ & 434.046 & 0.02530 \\
$\mathrm{H}$ & $2-4\left(\mathrm{H}_{\beta}\right)$ & 486.132 & 0.08419 \\
$\mathrm{H}$ & $2-3\left(\mathrm{H}_{\alpha}\right)$ & 656.280 & 0.4410 \\
\hline $\mathrm{He}$ & $6^{3} \mathrm{D}-2^{3} \mathrm{P}$ & 381.96 & 0.0589 \\
$\mathrm{He}$ & $3^{3} \mathrm{P}-2^{3} \mathrm{~S}$ & 388.865 & 0.09478 \\
$\mathrm{He}$ & $4^{1} \mathrm{P}-2^{1} \mathrm{~S}$ & 396.473 & 0.0717 \\
$\mathrm{He}$ & $5^{3} \mathrm{D}-2^{3} \mathrm{P}$ & 402.62 & 0.117 \\
$\mathrm{He}$ & $5^{3} \mathrm{~S}-2^{3} \mathrm{P}$ & 412.08 & 0.0430 \\
$\mathrm{He}$ & $6^{1} \mathrm{~S}-2^{1} \mathrm{P}$ & 416.897 & 0.0176 \\
$\mathrm{He}$ & $5^{1} \mathrm{D}-2^{1} \mathrm{P}$ & 438.793 & 0.0907 \\
$\mathrm{He}$ & $5^{1} \mathrm{~S}-2^{1} \mathrm{P}$ & 443.755 & 0.0313 \\
$\mathrm{He}$ & $4^{3} \mathrm{D}-2^{3} \mathrm{P}$ & 447.15 & 0.251 \\
$\mathrm{He}$ & $4^{3} \mathrm{~S}-2^{3} \mathrm{P}$ & 471.32 & 0.106 \\
$\mathrm{He}$ & $4^{1} \mathrm{D}-2^{1} \mathrm{P}$ & 492.193 & 0.202 \\
$\mathrm{He}$ & $3^{1} \mathrm{P}-2^{1} \mathrm{~S}$ & 501.568 & 0.1338 \\
$\mathrm{He}$ & $4^{1} \mathrm{~S}-2^{1} \mathrm{P}$ & 504.774 & 0.0655 \\
$\mathrm{He}$ & $3^{3} \mathrm{D}-2^{3} \mathrm{P}$ & 587.57 & 0.706 \\
$\mathrm{He}$ & $3^{1} \mathrm{D}-2^{1} \mathrm{P}$ & 667.815 & 0.638 \\
$\mathrm{He}$ & $3^{3} \mathrm{~S}-2^{3} \mathrm{P}$ & 706.53 & 0.278 \\
$\mathrm{He}$ & $3^{1} \mathrm{~S}-2^{1} \mathrm{P}$ & 728.135 & 0.181 \\
\hline \hline
\end{tabular}

excited state $p, n(p)$, is given by the model. Each state $p$ in this helium model is specified by the principal, the total electron angular, and the total electron spin quantum numbers. If photoexcitation is included, the temporal variation of the population density of an excited state $p$ can be described by a rate equation as

$$
\begin{aligned}
\mathrm{d} n(p) / \mathrm{d} t= & \sum_{q<p} C(q, p) n_{\mathrm{e}} n(q) \\
& +\sum_{q>p}\left\{F(q, p) n_{\mathrm{e}}+A(q, p)\right\} n(q) \\
& -\left\{\left(\sum_{q<p} F(p, q)+\sum_{q>p} C(p, q)+S(p)\right) n_{\mathrm{e}}\right. \\
& \left.+\sum_{q<p} A(p, q)\right\} n(p) \\
& +\delta_{p, 2{ }^{1} \mathrm{P}} I_{2^{1} \mathrm{P}} n\left(1^{1} \mathrm{~S}\right)+\delta_{p, 3^{1} \mathrm{P}} I_{3^{1} \mathrm{P}} n\left(1^{1} \mathrm{~S}\right) \\
& +\delta_{p, 4^{1} \mathrm{P}} I_{{ }^{1} \mathrm{P}} n\left(1^{1} \mathrm{~S}\right)+\cdots,
\end{aligned}
$$

where $n_{\mathrm{e}}$ is the electron density, $C(p, q)$ is the excitation rate coefficient for electron collisions from state $p$ to $q$, and $F(q, p)$ is the inverse de-excitation rate coefficient. $A(p, q)$ is the spontaneous transition probability from $p$ to $q$, and $S(p)$ is the ionization rate coefficient for state $p$. Each rate coefficient is a function of the electron temperature $T_{\mathrm{e}}$. The parameters $I_{2^{1} \mathrm{P}}, I_{3^{1} \mathrm{P}}$ and $I_{4^{1} \mathrm{P}}$ are photoexcitation rates from the ground state $1^{1} \mathrm{~S}$ to the $2^{1} \mathrm{P}, 3{ }^{1} \mathrm{P}$, and $4^{1} \mathrm{P}$ states per one atom, respectively [2]. The $\delta_{p, 2^{1} \mathrm{P}}, \delta_{p, 3{ }^{1} \mathrm{P}}$ and $\delta_{p, 4^{1} \mathrm{P}}$ are func- tions of two states; their values are 1 if the states are equal, and 0 otherwise. In the model, accurate cross sections for the electron impact transition calculated by the convergent close-coupling method [7-9] and the R-matrix with pseudostates method [10] are used.

According to the quasi-steady-state solution [3, 11], Eq. (1) is approximated to 0 for all the states except the ground state $1^{1} \mathrm{~S}$ and the metastable states $2^{1} \mathrm{~S}$ and $2^{3} \mathrm{~S}$ :

$$
\frac{\mathrm{d}}{\mathrm{d} t} n(p)=0
$$

Thus, instead of the coupled differential equations, Eq. (1), we have a set of coupled linear equations, Eq. (2).

Solving Eq. (1) with Eq. (2) yields the population density of an excited state $p$ in the form

$$
\begin{aligned}
n(p)= & r_{1}(p) n\left(1^{1} \mathrm{~S}\right) n_{\mathrm{e}}+r_{2}(p) n\left(2^{1} \mathrm{~S}\right) n_{\mathrm{e}} \\
& +r_{3}(p) n\left(2^{3} \mathrm{~S}\right) n_{\mathrm{e}}+r_{4}(p) n\left(1^{1} \mathrm{~S}\right) I_{2^{1} \mathrm{P}} \\
& +r_{5}(p) n\left(1^{1} \mathrm{~S}\right) I_{3^{1} \mathrm{P}}+r_{6}(p) n\left(1^{1} \mathrm{~S}\right) I_{4^{1} \mathrm{P}}+\cdots,
\end{aligned}
$$

where $r_{1}(p), r_{2}(p), r_{3}(p), r_{4}(p), r_{5}(p)$, and $r_{6}(p)$ are the population coefficients, each of which is a function of $n_{\mathrm{e}}$ and $T_{\mathrm{e}}$. The first three terms denote the conventional ionizing plasma component [3-6], while the other terms originate from photoexcitation [2]. By using the model, the parameters of $n_{\mathrm{e}}, T_{\mathrm{e}}, n\left(1^{1} \mathrm{~S}\right), n\left(2^{1} \mathrm{~S}\right), n\left(2^{3} \mathrm{~S}\right)$, and $I_{2^{1} \mathrm{P}}, I_{3^{1} \mathrm{P}}$, $I_{4^{1} \mathrm{P}}, \cdots$ can be determined experimentally from the intensities of the helium emission lines. 
In the present analysis of the emission intensities, we give the particle density estimated from the gas pressure to the helium atom density $n_{\mathrm{He}}\left(\equiv n\left(1^{1} \mathrm{~S}\right)\right)$ neglecting the small contribution of the hydrogen gas. The reason for this will be explained later. As the contributions of photoexcitation, only $1^{1} \mathrm{~S}$ to $3{ }^{1} \mathrm{P}$ and $4{ }^{1} \mathrm{P}$ are considered because emission lines originating from other singlet $\mathrm{P}$ states are not observed in our spectroscopic measurement.

\subsection{Collisional-radiative model for hydrogen atom}

In the present experiment, molecular processes which produce the excited state atoms are negligible as will be discussed later. We describe the collisional-radiative model for hydrogen atom without considering the molecular processes.

For atomic hydrogen, a model in which states are specified by the principal quantum numbers is used. Here $p$ denotes the principal quantum number. If photoexcitation from the ground state is taken into account, the population density of an excited state $p$ is written as follows $[1,12]$ :

$$
\begin{aligned}
n(p)= & R_{1}(p) n(1) n_{\mathrm{e}}+R_{\alpha}(p) n(1) I_{2} \\
& +R_{\beta}(p) n(1) I_{3}+R_{\gamma}(p) n(1) I_{4}+\cdots,
\end{aligned}
$$

where $R_{1}(p), R_{\alpha}(p), R_{\beta}(p)$, and $R_{\gamma}(p)$ are the population coefficients, each of which are respectively a function of $n_{\mathrm{e}}$ and $T_{\mathrm{e}}$. The first term denotes the conventional ionizing plasma component $[3,12]$. The other terms originate from photoexcitation. The parameters $I_{2}, I_{3}, I_{4}, \cdots$ are photoexcitation rates from the ground state to the excited states $p=2,3,4, \cdots$ per atom, respectively [1]. In the present study, the original code in Refs. $[1,12]$ is revised to include recent reliable electron impact excitation rate coefficients calculated by the method of R-matrix with pseudostates [13]. The rate coefficients are given for transitions among $p=1-5$ states in the electron temperature range of $0.5-25.0 \mathrm{eV}$. The corresponding rate coefficients in the original code are replaced with the new data.

To calculate $I_{2}, I_{3} I_{4}, \cdots$ in Eq. (4), the iterative selfconsistent method [1] is applied. When $T_{\mathrm{e}}$ and $n_{\mathrm{e}}$, the atomic hydrogen density $n_{\mathrm{H}}(\equiv n(1))$, and the line profile functions of the Lyman series are given, the following algorithm is applied to the radiation trapping analysis:

1. Divide the space into cubic cells of linear dimension $\Delta l$.

2. For each cell, give $n_{\mathrm{e}}, T_{\mathrm{e}}, n(1)$, and the line profile function $g_{p}(v)$ for the transition from the upper state $p$ to the ground state. The line profile function $g_{p}(v)$ is defined so that the probability of emission in the frequency interval $v \sim v+\mathrm{d} v$ is $g_{p}(v) \mathrm{d} v$. Set the frequency interval, $\Delta v$, for the subsequent calculation of emission and absorption. For each cell, calculate the absorption coefficient $\kappa_{p}(v)$ which is given by

$$
\kappa_{p}(v)=\frac{B(1, p) n(1) h v g_{p}(v)}{c},
$$

where $B(1, p)$ is the Einstein B coefficient and $c$ is the speed of light.

3. Compute the excited-state population distribution for each cell using Eq. (4) without considering the photoexcitation terms.

4. Compute the emission intensity radiated in each cell. The power radiated by any cell per unit frequency is given by

$$
\varepsilon_{p}(v)=A(p, 1) n(p) g_{p}(v) h v \Delta V,
$$

where $\Delta V$ is the volume of the cell $\left(=(\Delta l)^{3}\right)$. We assume isotropic photon emission and complete frequency redistribution, so the same $g_{p}(v)$ is used for absorption and emission.

Next, compute the spread of the emitted photons by considering absorption in other cells using the absorption coefficient obtained in step 2. At a cell whose distance from the source is $r$, the energy density per unit frequency $\rho_{p}(v)$ is calculated by

$\rho_{p}(v)=\frac{\varepsilon_{p}(v)}{c} \frac{1}{4 \pi r^{2}} \exp \left[-\int_{\text {source }}^{r} \kappa_{p}(v) \mathrm{d} r\right]$,

where the integration in the exponential is over the line-of-sight from the source to the cell, and is performed numerically.

5. The total energy density per unit frequency, $\rho_{p}^{\text {total }}(v)$ (see Fig. 11.), is calculated at each cell by summing the contributions of photons from all cells. The parameter $I_{p}$ in Eq. (4) is numerically calculated as

$$
I_{p} \equiv B(1, p) \int \rho_{p}^{\text {total }}(v) g_{p}(v) \mathrm{d} v
$$

Taking into account photoexcitation, compute the population distribution for each cell using Eq. (4).

6. Iterate steps 4 and 5 until the above values converge.

\section{Results and Discussion}

Figure 3 shows that the intensities of helium and hydrogen lines differ in their radial dependence. To precisely determine $T_{\mathrm{e}}$ and $n_{\mathrm{e}}$, the Balmer $\gamma$ line of atomic hydrogen, whose line intensity is little affected by photoexcitation from the ground state atom, is added to the analysis of the helium lines; the density of the ground state hydrogen atom $n_{\mathrm{H}}$ is simultaneously determined in addition to the parameters $n_{\mathrm{e}}, T_{\mathrm{e}}, I_{3^{1} \mathrm{P}}, I_{4^{1} \mathrm{P}}, n\left(2^{1} \mathrm{~S}\right)$, and $n\left(2^{3} \mathrm{~S}\right)$. The Balmer $\gamma$ line intensity is calculated using the collisional-radiative model for atomic hydrogen, ignoring photoexcitation from the ground state. By changing the values of each parameter, we determined the best set of parameter values numerically by least-squares fitting. $n_{\mathrm{He}}$ and $n_{\mathrm{H}}$ were assumed to be spatially uniform. 
Figure 4 shows the population distribution of the singlet and triplet states of helium atoms at $R=1.75 \mathrm{~cm}$ in Fig. 3(b). The population density calculated with the optimized parameters is also shown. The experimentally obtained population was reproduced well by least-squares fitting. Most of the population of $3{ }^{1} \mathrm{P}$ and $4{ }^{1} \mathrm{P}$ is found to be produced by photoexcitation from the ground state. The populations of other states are produced mainly by the first term on the right-hand side of Eq. (3). Photoexcitation to the $3^{1} \mathrm{P}$ and $4^{1} \mathrm{P}$ states has little effect on the population density of other states because of the low electron density in the present experiment. Photoexcitation to singlet $\mathrm{P}$ states higher than $4^{1} \mathrm{P}$ can obviously be neglected in the present analysis. Similarly, the $2^{1} \mathrm{P}$ produced by photoexcitation decays into $1^{1} \mathrm{~S}$ and $2^{1} \mathrm{~S}$ by a radiative transition. The subsequent electron impact excitation from ${ }^{1} \mathrm{~S}$ and $2^{1} \mathrm{~S}$ is included in the terms $r_{1}(p) n\left(1{ }^{1} \mathrm{~S}\right) n_{\mathrm{e}}$ and $r_{2}(p) n\left(2^{1} \mathrm{~S}\right) n_{\mathrm{e}}$ in Eq. (3). Figure 5 shows the Balmer $\gamma$ line intensity calculated with the optimized parameters. The experimental intensity is reproduced well. Figure 6 shows $T_{\mathrm{e}}$ and $n_{\mathrm{e}}$. The value determined for $n_{\mathrm{H}}$ is $1.0 \times 10^{14} \mathrm{~cm}^{-3}$.

In hydrogen plasmas, excited state hydrogen atoms can be produced by the electron impact dissociative excitation of molecular hydrogen [14-16]. In the present experiment, the molecular hydrogen density $n_{\mathrm{H}_{2}}$ estimated from the intensity of the Fülcher band in Fig. 2(b) is $7.6 \times 10^{13} \mathrm{~cm}^{-3}$. A corona model for the Fülcher band [17] that includes an excitation cross section $\left(X^{1} \Sigma_{g}^{+} \rightarrow d^{3} \Pi_{u}\right)$ from Ref. [18] is used in this estimation. Figure 7 shows

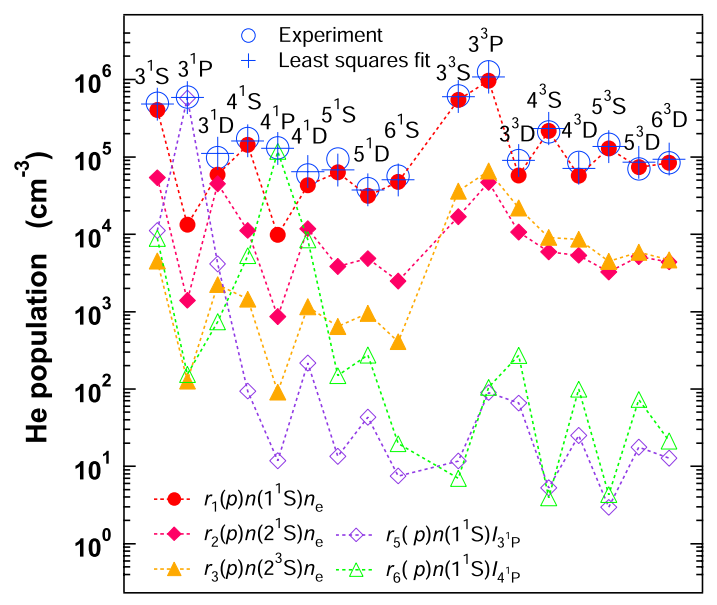

Fig. 4 Population distribution of singlet and triplet states at $R=1.75 \mathrm{~cm}$. Open circles: spectroscopic measurement; plus signs: result of the least-squares fit calculated using Eq. (3) with optimized values of $n_{\mathrm{e}}, T_{\mathrm{e}}, I_{3^{1} \mathrm{P}}, I_{4^{1} \mathrm{P}}$, $n\left(2^{1} \mathrm{~S}\right), n\left(2^{3} \mathrm{~S}\right)$, and $n_{\mathrm{H}}$. The contributions of each term of Eq. (3) are also shown. Closed circles: $r_{1}(p) n\left(1^{1} \mathrm{~S}\right) n_{\mathrm{e}}$; closed diamonds: $r_{2}(p) n\left(2^{1} \mathrm{~S}\right) n_{\mathrm{e}}$; closed triangles: $r_{3}(p) n\left(2^{3} \mathrm{~S}\right) n_{\mathrm{e}}$; open diamonds: $r_{5}(p) n\left(1^{1} \mathrm{~S}\right) I_{3^{1} \mathrm{P}}$; open triangles: $\quad r_{6}(p) n\left(1^{1} \mathrm{~S}\right) I_{4^{1} \mathrm{P}}$. The uncertainties of $r_{2}(p) n\left(2^{1} \mathrm{~S}\right) n_{\mathrm{e}}$ and $r_{3}(p) n\left(2^{3} \mathrm{~S}\right) n_{\mathrm{e}}$ may be large because their contributions are small. the excitation rate coefficient from the ground state atomic hydrogen and the production rate coefficient of excited atoms from molecular hydrogen calculated by a code in Ref. [16] for $T_{\mathrm{e}}=3.0 \mathrm{eV}$. It is seen that the rate coefficient of dissociative excitation is much smaller than that of the excitation from the ground state of atomic hydrogen. In addition, $n_{\mathrm{H}_{2}}$ is lower than $n_{\mathrm{H}}$. Thus, the dissociative excitation of molecular hydrogen is negligible for the production of excited hydrogen atoms in the experiment presented here. This conclusion is supported by the upper limit of $n_{\mathrm{H}_{2}}$ estimated from the gas pressure with the flow

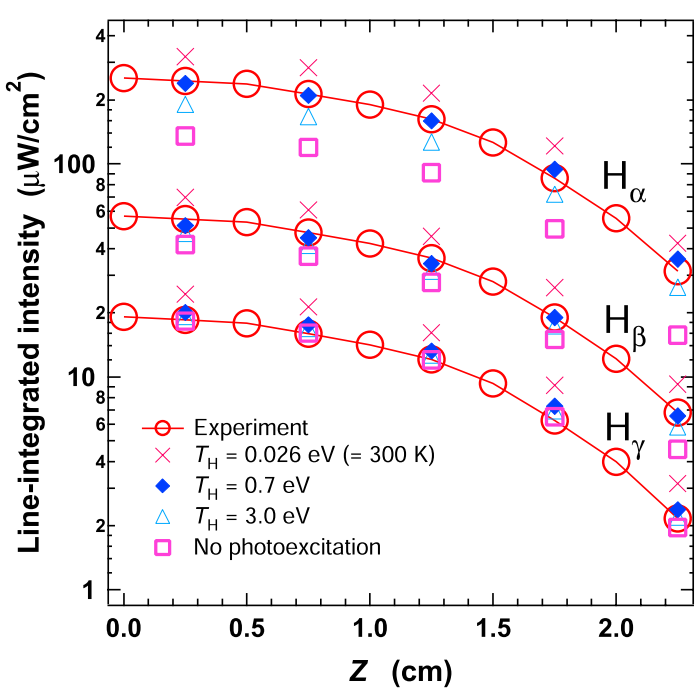

Fig. 5 Line-of-sight integrated Balmer series line intensities. Open circles: spectroscopic measurement; crosses: calculated values with $T_{\mathrm{H}}=0.026 \mathrm{eV}(=300 \mathrm{~K})$; closed diamonds: calculated values with $T_{\mathrm{H}}=0.7 \mathrm{eV}$; open triangles: calculated values with $T_{\mathrm{H}}=3 \mathrm{eV}$; open squares: calculated values ignoring photoexcitation. $n_{\mathrm{H}}=1.0 \times$ $10^{14} \mathrm{~cm}^{-3}$.

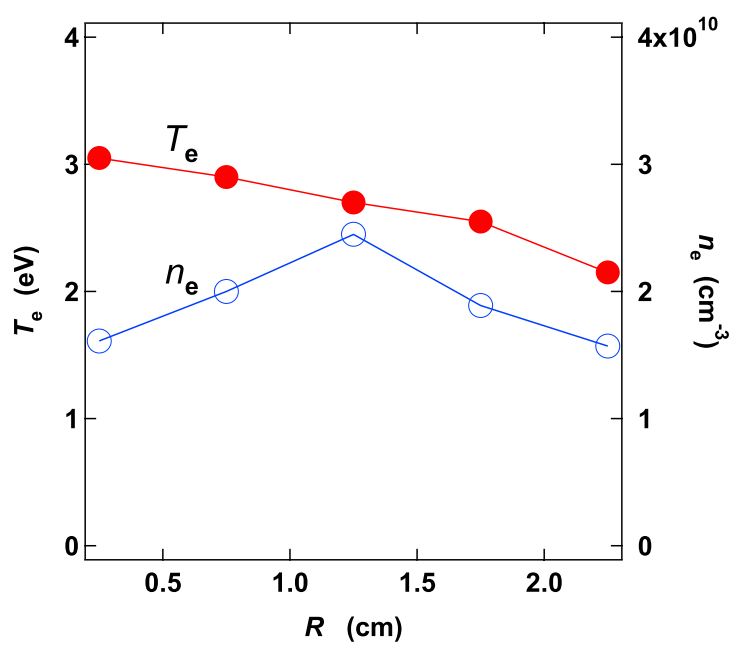

Fig. $6 T_{\mathrm{e}}$ and $n_{\mathrm{e}}$ determined by the spectroscopic method. Closed circles: $T_{\mathrm{e}}$; open circles: $n_{\mathrm{e}}$. 
ratio of helium and hydrogen gas. The production of excited atoms by molecular assisted recombination $[19,20]$, which is calculated using a collisional-radiative model for molecular hydrogen $[17,20]$, is also found to be negligible. The ratio of hydrogen atoms reflected at the glass wall as atoms without forming molecules is expected to be large from $n_{\mathrm{H}} / n_{\mathrm{H}_{2}}$ [21].

As shown in Fig. 5, the experimental Balmer $\alpha$ and $\beta$ lines are not reproduced by the collisional-radiative model in which photoexcitation from the ground state is neglected. This discrepancy strongly suggests the influence of radiation trapping of atomic hydrogen Lyman series emission. In addition to the parameters determined above, by assuming that the line profile function of the

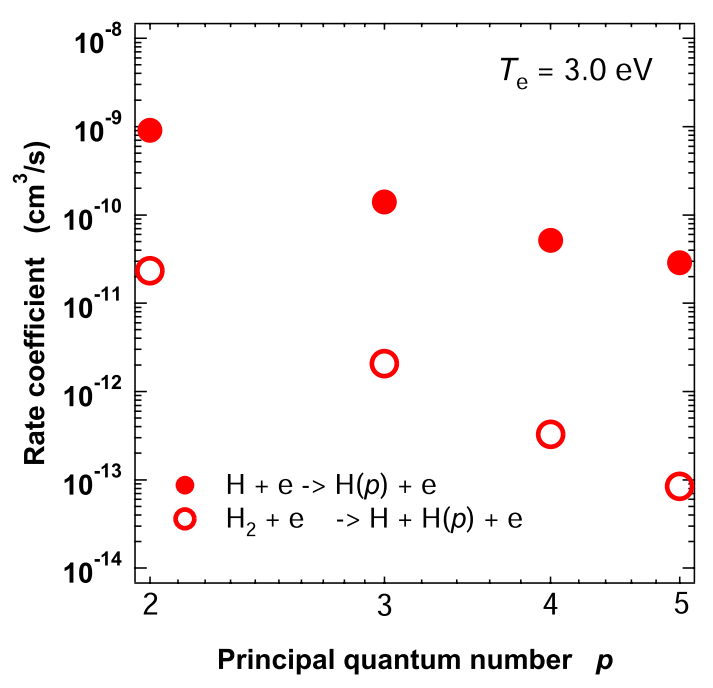

Fig. 7 Rate coefficient calculated for $T_{\mathrm{e}}=3.0 \mathrm{eV}$. Closed circles: electron impact excitation of atomic hydrogen from the ground state to the excited state $p$; open circles: electron impact dissociative excitation of molecular hydrogen that produces the excited state $p$ atom.

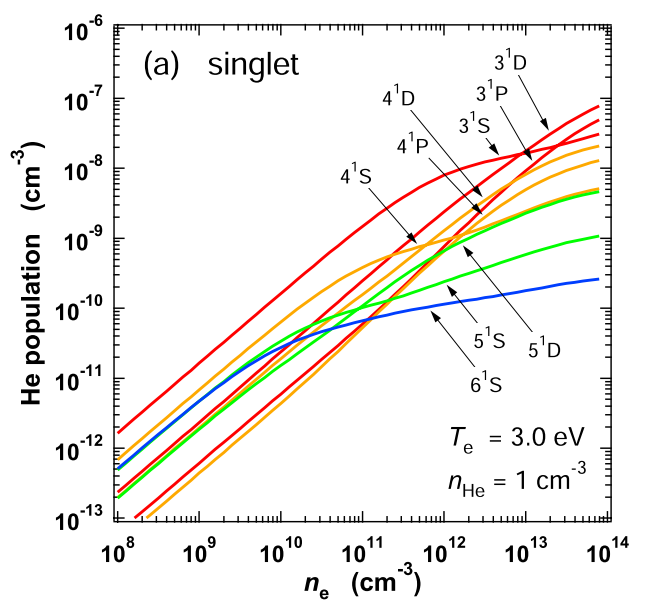

Lyman series $g_{p}(v)$ is given by the Doppler broadening with a Maxwellian velocity distribution, the atomic hydrogen temperature $T_{\mathrm{H}}$ can be evaluated using the iterative collisional-radiative model of atomic hydrogen [1]. We assume $T_{\mathrm{H}}$ is spatially uniform and the plasma is uniform along the glass cylinder's axis. We calculate the intensities of the Balmer $\alpha$ and $\beta$ lines with the iterative collisionalradiative model by varying $T_{\mathrm{H}}$. Figure 5 shows the calculated intensities. The upper limit of $T_{\mathrm{H}}$ is about $3 \mathrm{eV}$ for Franck-Condon atoms produced by molecular dissociation, and the lower limit of $T_{\mathrm{H}}$ is room temperature. As shown in Fig. $5, T_{\mathrm{H}}=0.7 \mathrm{eV}$ reproduces the experimental intensity of the Balmer $\alpha$ and $\beta$ lines well.

For helium atom in the present study, as shown in Fig. $4, r_{1}(p) n\left(1^{1} \mathrm{~S}\right) n_{\mathrm{e}}$ is dominant in the terms on the righthand side of Eq. (3) except for the singlet $\mathrm{P}$ states, which are affected by photoexcitation. In this study, the determined $n_{\mathrm{e}}$ and $T_{\mathrm{e}}$ range from $1.6 \times 10^{10}$ to $2.5 \times 10^{10} \mathrm{~cm}^{-3}$ and 2.2-3.1 eV, respectively. Figures 8 (a) and 8 (b) show the $n_{\mathrm{e}}$ dependence of $r_{1}(p) n\left(1{ }^{1} \mathrm{~S}\right) n_{\mathrm{e}}$ calculated for $T_{\mathrm{e}}=$ $3.0 \mathrm{eV}$. Figures 9 (a) and 9 (b) show the $T_{\mathrm{e}}$ dependence of this term calculated for $n_{\mathrm{e}}=2 \times 10^{10} \mathrm{~cm}^{-3}$. For hydrogen atoms, Figs. 10 (a) and 10 (b) show the $n_{\mathrm{e}}$ and $T_{\mathrm{e}}$ dependence, respectively, of $R_{1}(p) n(1) n_{\mathrm{e}}$ in Eq. (4). From Figs. 8, 9 and 10, we can see that the difference in radial dependence between the helium and hydrogen lines in Fig. 3 (b) comes mainly from the $T_{\mathrm{e}}$ dependence of the population densities of both atoms. In the present range of $n_{\mathrm{e}}$ and $T_{\mathrm{e}}$, we can simplify the least-squares fit as follows: $n_{\mathrm{e}}$ is determined from the relative population distribution of excited states of helium atom other than the singlet $\mathrm{P}$ states, because the relative population distribution changes only slightly with $T_{\mathrm{e}}$ in the present range of $T_{\mathrm{e}} . T_{\mathrm{e}}$ is determined from the absolute value of the population of excited helium atoms by using $n_{\mathrm{e}}$ and $n\left(1^{1} \mathrm{~S}\right)$. As mentioned before, we assign the particle density estimated from the gas pressure to $n\left(1^{1} \mathrm{~S}\right)$ because both $T_{\mathrm{e}}$ and $n\left(1^{1} \mathrm{~S}\right)$ cannot be

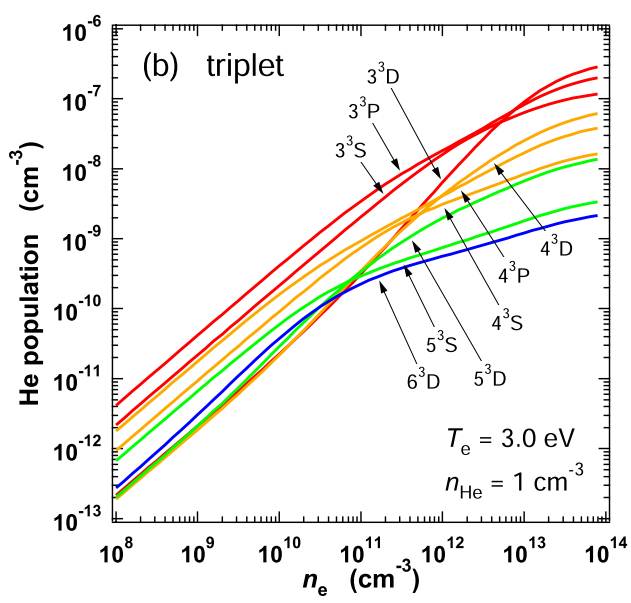

Fig. 8 Electron density dependence of $r_{1}(p) n\left(1^{1} \mathrm{~S}\right) n_{\mathrm{e}}$ in Eq. (3) calculated for $T_{\mathrm{e}}=3.0 \mathrm{eV}$ and $n\left(1^{1} \mathrm{~S}\right)=1 \mathrm{~cm}^{-3}$. (a) Singlet states. (b) Triplet states. 

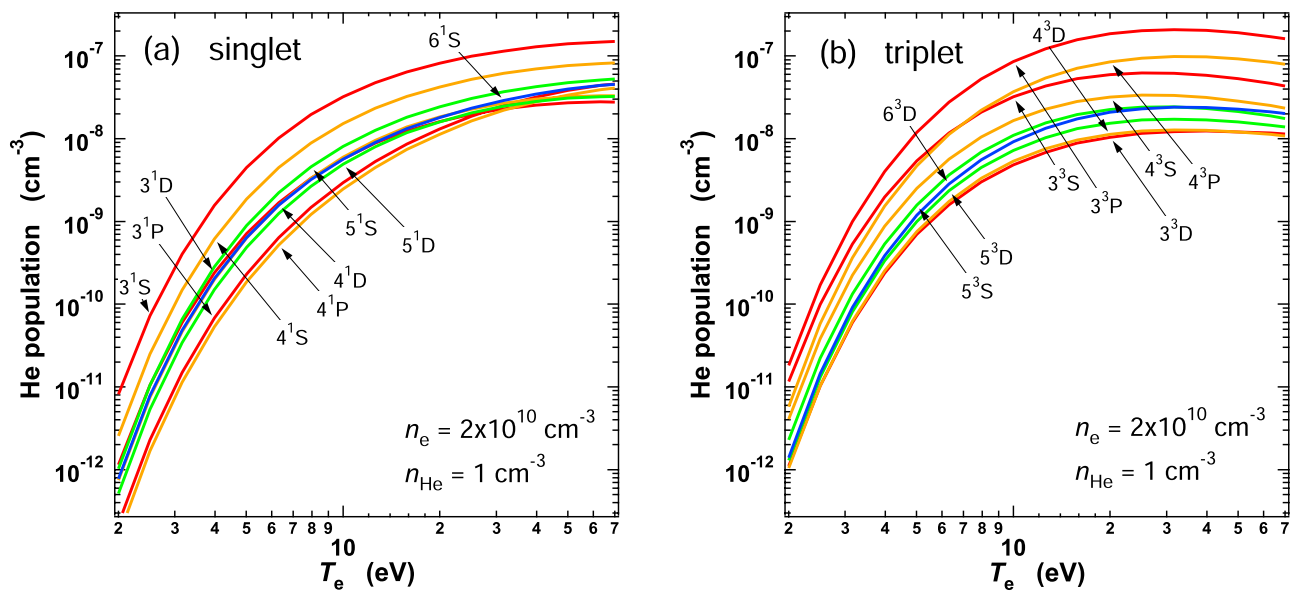

Fig. 9 Electron temperature dependence of $r_{1}(p) n\left(1^{1} \mathrm{~S}\right) n_{\mathrm{e}}$ in Eq. (3) calculated for $n_{\mathrm{e}}=2 \times 10^{10} \mathrm{~cm}^{-3}$ and $n\left(1{ }^{1} \mathrm{~S}\right)=1 \mathrm{~cm}^{-3}$. (a) Singlet states. (b) Triplet states.
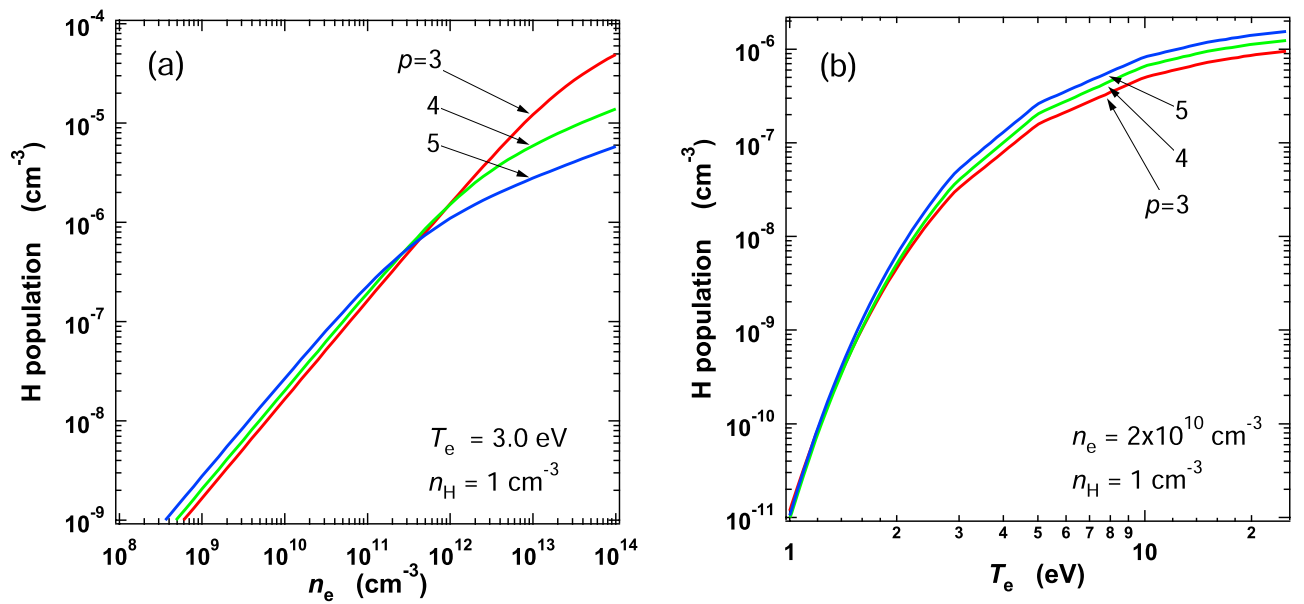

Fig. $10 R_{1}(p) n(1) n_{\mathrm{e}}$ in Eq. (4). $n(1)=1 \mathrm{~cm}^{-3}$. (a) Electron density dependence at $T_{\mathrm{e}}=3.0 \mathrm{eV}$. (b) Electron temperature dependence at $n_{\mathrm{e}}=2 \times 10^{10} \mathrm{~cm}^{-3}$.

determined from the absolute value of the population. $n_{\mathrm{H}}$ is determined from the absolute intensity of Balmer $\gamma$ of the hydrogen atom by using $n_{\mathrm{e}}$ and $T_{\mathrm{e}}$.

We estimate the uncertainties of the obtained parameters from Figs. 8, 9, and 10. The uncertainty of each local emission line intensity of helium atoms in Fig. 3 (b) is about $10 \%$. From this uncertainty, the uncertainty of $n_{\mathrm{e}}$, $\delta n_{\mathrm{e}}$, in Fig. 6 is estimated to be about $0.2 \times 10^{10} \mathrm{~cm}^{-3}$ by using Fig. 8. The uncertainty of $n\left(1^{1} \mathrm{~S}\right), \delta n\left(1^{1} \mathrm{~S}\right)$ is about $0.4 \times 10^{15} \mathrm{~cm}^{-3}$. The uncertainty of $T_{\mathrm{e}}, \delta T_{\mathrm{e}}$, in Fig. 6 is estimated to be about $0.1 \mathrm{eV}$ from $\delta n_{\mathrm{e}}$ and $\delta n\left(1^{1} \mathrm{~S}\right)$ by using Fig. 9. The uncertainty of the line-integrated intensity of Balmer $\gamma$ is about $3 \%$. The uncertainty of $n_{\mathrm{H}}, \delta n_{\mathrm{H}}$, is estimated to be about $0.2 \times 10^{14} \mathrm{~cm}^{-3}$ by using $\delta n_{\mathrm{e}}$ and $\delta T_{\mathrm{e}}$, and the uncertainty of the Balmer $\gamma$ intensity. The uncertainty of $T_{\mathrm{H}}$, which is estimated from the uncertainties of the above parameters, is about $0.2 \mathrm{eV}$.

Table 2 lists the reciprocal of the absorption coefficients at the central frequencies of the spectral lines $l_{M}$
Table 2 Mean free paths for the absorption of photons at the central frequencies of the Lyman spectral lines. $T_{\mathrm{H}}$ and $n_{\mathrm{H}}$ are $0.7 \mathrm{eV}$ and $1.0 \times 10^{14} \mathrm{~cm}^{-3}$, respectively.

\begin{tabular}{ll}
\hline \hline $\mathrm{L}_{\alpha}$ & $0.15 \mathrm{~cm}$ \\
$\mathrm{~L}_{\beta}$ & $0.96 \mathrm{~cm}$ \\
$\mathrm{~L}_{\gamma}$ & $2.8 \mathrm{~cm}$ \\
$\mathrm{~L}_{\delta}$ & $5.9 \mathrm{~cm}$ \\
\hline
\end{tabular}

calculated for $T_{\mathrm{H}}=0.7 \mathrm{eV}$ and $n_{\mathrm{H}}=1.0 \times 10^{14} \mathrm{~cm}^{-3}$, which corresponds to the mean free paths for the absorption of photons at that frequency. The lower of the two values $l_{M} / 10$ and $0.5 \mathrm{~cm}$ was assigned to the edge length of the cubic cells $\Delta l$ in the iterative calculation of each Lyman line. The frequency interval of each Lyman line $\Delta v$ was set to FWHM/10 of the line profile function $g_{p}(v)$ in Eq. (5) as shown in Fig. 11. The total energy density 


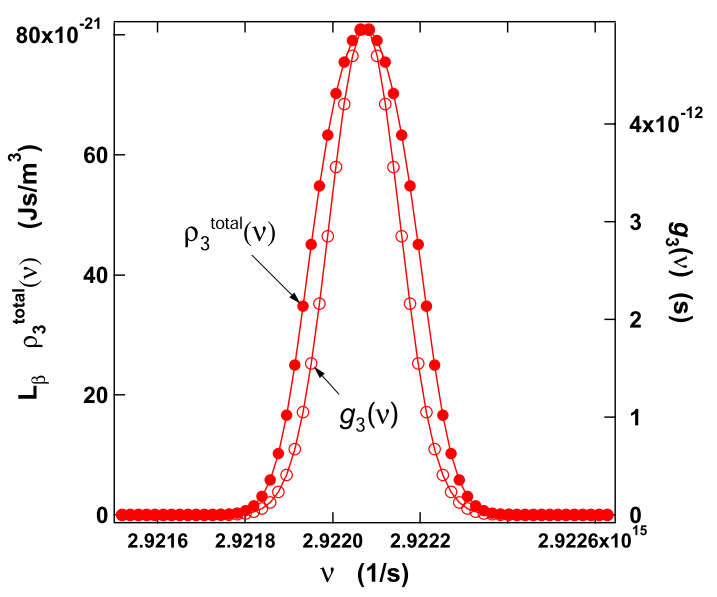

Fig. 11 The total energy density per unit frequency $\rho_{3}^{\text {total }}(v)$ in Eq. (8) at $R=0 \mathrm{~cm}$ obtained after iteration. Line profile function $g_{3}(v)$ in Eqs. (5), (6), and (8) is also shown. $T_{\mathrm{H}}$ and $n_{\mathrm{H}}$ are $0.7 \mathrm{eV}$ and $1.0 \times 10^{14} \mathrm{~cm}^{-3}$, respectively. Closed circles: $\rho_{3}^{\text {total }}(v)$; open circles: $g_{3}(v)$.

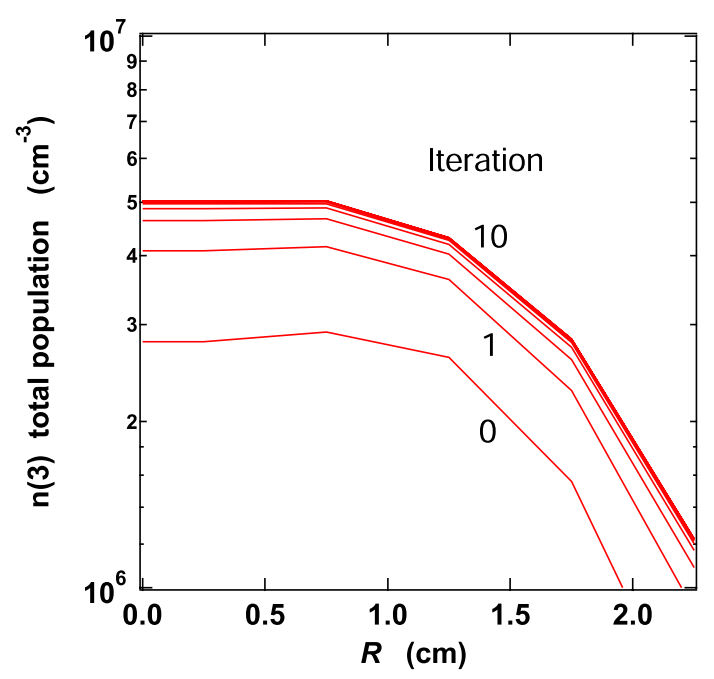

Fig. 12 Population of $n(3)$ in the iteration process. Line labeled "0" corresponds to the calculation without photoexcitation. $T_{\mathrm{H}}$ and $n_{\mathrm{H}}$ are $0.7 \mathrm{eV}$ and $1.0 \times 10^{14} \mathrm{~cm}^{-3}$, respectively.

per unit frequency $\rho_{3}^{\text {total }}(v)$ in Eq. (8) at $R=0 \mathrm{~cm}$ obtained after iteration is shown in Fig. 11. Figure 12 shows the characteristic of the iteration. The calculation converges in approximately three iterations.

\section{Conclusions}

We determine $T_{\mathrm{e}}, n_{\mathrm{e}}, n_{\mathrm{H}}$, and $T_{\mathrm{H}}$ in a helium-hydrogen RF plasma from the visible emission line intensity of both atoms. At the same time, we understand that singlet $\mathrm{P}$ state helium atoms are produced predominantly by photoexcitation, and the population of the upper state of the Balmer $\alpha$ line is strongly influenced by radiation trapping. Radiation trapping usually makes it difficult to analyze the intensity of emission lines. However, in the present study, detailed analysis of the effect allows us to determine $T_{\mathrm{H}}$. For plasmas that have higher electron temperatures and densities, e.g., fusion plasmas, the helium density can be determined from the emission line intensities in addition to the parameters determined in this study.

In the present plasma, molecular processes that produce excited state hydrogen atom are negligible. When these processes become significant, the iterative collisional-radiative model can easily be extended to include them. The Balmer series lines emitted from higher states, $\mathrm{H}_{\delta}, \mathrm{H}_{\epsilon}, \cdots$, become more important for determining the densities of atoms and molecules.

\section{Acknowledgments}

We wish to thank students of Shinshu University, K. Fukutomi, F. Yokoyama, T. Oda, Y. Kambe, and S. Kakura for their assistance during the experimental work. We also thank H. Hara for technical support, and former graduate students, S. Nakauchi, K. Kawasaki, H. Fujiwara, M. Shinohara, E. Kusunoki, K. Shiraki, M. Tsujii, Y. Suzawa, T. Kubo, M. Ueda for help in building up the experimental set up and developing the codes. We are grateful to K. Fujii for supplying information on cross section data. The research was partially supported by Grant-in-Aid for Scientific Research (C), No.15540470 and No.21540508, from the Japan Society for the Promotion of Science, and by the NIFS Collaborative Research Program. We would like to thank the referee for thoughtful suggestions which helped us to improve our paper.

[1] K. Sawada, J. Plasma Phys. 72, 1025 (2006).

[2] K. Sawada, Y. Yamada, T. Miyachika, N. Ezumi, A. Iwamae and M. Goto, Plasma Fusion Res. 5, 001 (2010).

[3] T. Fujimoto, Plasma Spectroscopy (The International Series of Monographs on Physics, 123, Oxford University Press, 2004).

[4] T. Fujimoto, JQSRT 21, 439 (1978).

[5] M. Goto, JQSRT 76, 331 (2003).

[6] M. Goto, S. Morita, K. Sawada, T. Fujimoto and S. Yamamoto, Phys. Plasmas 10, 1402 (2003).

[7] D.V. Fursa and I. Bray, Phys. Rev. A 52, 1279 (1995).

[8] I. Bray and D.V. Fursa, J. Phys. B 28, L197 (1995).

[9] D.V. Fursa and I. Bray, J. Phys. B 30, 757 (1997).

[10] K. Bartschat, J. Phys. B 31, L469 (1998).

[11] K. Sawada and T. Fujimoto, Phys. Rev. E 49, 5565 (1994).

[12] T. Fujimoto, S. Miyachi and K. Sawada, Nucl. Fusion 28, 1255 (1988).

[13] H. Anderson, C.P. Ballance, N.R. Badnell and H.P. Summers, J. Phys. B 33, 1255 (2000); Anderson, Ballance, Badnell and Summers, J. Phys. B 35, 1613 (2002).

[14] T. Fujimoto, K. Sawada and K. Takahata, J. Appl. Phys. 66, 2315 (1989).

[15] T. Fujimoto, K. Sawada, K. Takahata, K. Eriguchi, H. Suemitsu, K. Ishii, R. Okasaka, H. Tanaka, T. Maekawa, Y. Terumichi and S. Tanaka, Nucl. Fusion 29, 1519 (1989).

[16] K. Sawada, K. Eriguchi and T. Fujimoto, J. Appl. Phys. 73, 8122 (1993). 
[17] K. Sawada, Report on Grant-in-Aid for scientific research No.15540470 (2007) [in Japanese].

[18] G.R. Möhlmann and F.J. de Heer, Chem. Phys. Letters 43, 240 (1976).

[19] S.I. Krasheninnikov, A.Yu. Pigarov and D.J. Sigmar, Phys.
Lett. A 214, 285 (1996).

[20] K. Sawada and T. Fujimoto, Contrib. Plasma Phys. 42, 603 (2002).

[21] B.J. Wood and H. Wise, J. Phys. Chem. 66, 1049 (1962). 